for prescribed variables in the model and assessed for quality using the EPI-review tool. The review included an analysis of methodologies, questionnaires, recruitment strategies and raw data used in and generated by previous studies.

The TWG found approximately $50 \%$ of the data needed for use in the MOT study. Methodological issues with data collection activities were identified, key questions used in population-based studies were modified and reconstructed, and formal population size estimations were recommended.

The review generated substantive recommendations to enhance future data collection activities and improve programming. Stakeholder awareness about limitations of available data and the types of studies/data needed to help better understand the epidemic and determine appropriate responses. Periodic reviews of data availability and quality are critical to knowing your epidemic and the most appropriate response.

The EPI- review tool increased stakeholder's awareness about the limitations of the available data and the types of studies/data needed to help better understand the epidemic and determine appropriate responses. This capacity building effort generated substantial recommendations to enhance future data collection activities. The tool saved time and effort at the country level by focusing on the data needed to conduct the MOT.

\section{P3.231 STUDY DRUG INTERRUPTION AMONG HIV SERODISCORDANT COUPLES IN PARTNERS PREP STUDY, THIKA-KENYA}

doi:10.1136/sextrans-2013-051184.0688

'P A Mogere, 'E Irungu, 'S Mbaire, 1,2 $\mathrm{G}$ Mugendi, 'N Njuguna, 1,3K Ngure, ${ }^{1,4} \mathrm{~N}$ Mugo. 'Kenyatta National Hospital, Nairobi, Kenya; 'University of Nairobi, Nairobi, Kenya; ${ }^{3} J o m o$ Kenyatta University, Nairobi, Kenya; ${ }^{4}$ University of Washington, Seattle, WA, United States

Background For successful implementation of PrEP as a HIV prevention strategy, participant's adherence to the intervention is vital. Frequent interruption of study product, impacts its effectiveness negatively. We documented the frequency and causes of study drug interruptions among participants enrolled in the Partners PrEP Study, Thika-Kenya

Methods Between October 2008 and November 2010, we enrolled 496 HIV serodiscordant couples who were followed up to 36 months. We categorised time off study drug into two; protocol-defined, in which the parameters of withholding study drug was clearly outlined in the study protocol and participant-initiated interruption where the participant opted to stop taking study drug. Data on study drug interruptions were captured on monthly basis and documented on specific case report forms in pharmacy.

Results We reported 152 cases of study drug interruptions, these were contributed by 128 participants. Sixty four per cent of HIV-1 uninfected participants who recorded study drug interruption were male, median age for both gender was 31.8 (IOR 26.5, 37.4) years. Sixty five $(43 \%)$ of reported drug interruptions were participant initiated, cited due to marital disharmony 34 (22\%), fatigue and loss of interest $24(16 \%)$, perceived side effects in $7(5 \%)$. The median time off study product was 90 (IOR 28, 268) days. Age, gender and education were not associated with participant-initiated interruptions. Pregnancy and possible seroconversion were some of protocol defined reasons to stop study drug.

Conclusions Marital disharmony and loss of interest were two of the most common reasons for participant initiated interruption of study drug. Going forward, psychosocial support and continuous adherence counselling should be part of the package for successful implementation of PrEP for HIV prevention.

\section{P3.232 PREVALENCE OF MYCOPLASMA GENITALIUM AMONG WOMEN ATTENDING SEXUALLY TRANSMITTED INFECTION CLINIC IN KUMASI, GHANA}

doi:10.1136/sextrans-2013-051184.0689

${ }^{1}$ T Agyarko-Poku, ${ }^{2} Y$ Adu Sarkodie, ${ }^{2}$ E Hene Frimpong. ${ }^{1}$ Suntreso Government Hospital, Ghana Health Services, Kumasi, Ghana; 2 School of Medical Sciences, Kwame Nkrumah University of Science and Technology, Kumasi, Ghana

Background Mycoplasma genitalium (MG) has been identified over the past decade as an aetiological agent of non-gonococci cervicitis in women. A multinational study in among female sex workers in West Africa which included Kumasi and Accra Ghana established Mycoplasma genitalium prevalence of $26.3 \%$. The literature is however silent on prevalence of MG among women in the general population in Ghana. This study sought to determine the current state of affair in women patronising Sexually Transmitted Infection (STI) Clinic in Kumasi, Ghana.

Methods Specimens for DNA polymerase chain reaction (PCR) determination, were collected from the vagina and the cervix of 300 women: 150 sex workers (SW) and 150 non-sex workers (NSW), attending Suntreso STI Clinic in Kumasi for the first time, with complaint of vaginal discharge. Socio demographic characteristics of the women, symptoms and signs were recorded. Associations of factors with Mycoplasma genitalium were recorded and adjusted for other risk factors.

Results Ten (10) out of the 300 women representing 3.3\% (10/300), were found to have Mycoplasma genitalium; $p=0.000, \mathrm{OR}=0.26$, $95 \% \mathrm{CI}=0.07-0.87, X^{2}=0.27$. Prevalence of Mycoplasma genitalium in female sex workers was higher $(4.7 \%, 7 / 150)$ than non-sex workers $(2.0 \%, 3 / 150)$. Younger age (15-29 years, 5.4\%, 9/167) was found to be the strongest predictor of Mycoplasma genitalium.

Conclusion The study confirms Mycoplasma genitalium as an aetiological agent of vaginal discharge in women in Kumasi Ghana, conforming to other studies in West Africa with lower prevalence rate. It is possible that the actual rate, in the general population may be low as indicated by even lower rate among high-risk group like sex workers. Further study with larger sample size at the population level is required to guide the course of management.

\section{P3.233 GENITAL TRACT ABNORMALITIES IN HIV-TB CO-INFECTED WOMEN INITIATING ANTIRETROVIRAL THERAPY (ART)}

doi:10.1136/sextrans-2013-051184.0690

K Naidoo, M Naidoo, S Gengiah, N Nkupiso, C Singh, K Leask, A BM Kharsany. Caprisa, Durban, South Africa

Background HIV infected women have an increased risk of acquiring genital tract infections and progression of pre-malignant cervical lesions. We describe the prevalence and incidence of genital tract abnormalities in TB-HIV co-infected women initiating ART(Antiretroviral therapy).

Methods We conducted a retrospective study among 750 ambulant TB-HIV co-infected women initiating ART in Durban, between 2004 and 2011. All patients received sexual reproductive health services including a Papanicolaou (Pap) smear examination; and screening; diagnosis and management of sexually transmitted infections (STIs). Pap smear reporting included the Bethesda classification for endocervical abnormalities, and STI screening for Human papilloma virus (HPV), trichomonas vaginalis, bacterial vaginosis (BV) and candidiasis.

Results Baseline pap smears were obtained before or up to 6 months post-ART initiation in 750 women; mean (standard deviation(SD)) age 34.2 (8.0) years; mean CD4+ count 181.4 (SD 178.5) cells $/ \mathrm{mm}^{3}$ and median log viral load 4.4 (IOR 2.6) copies $/ \mathrm{ml}$. Prevalence of genital tract abnormalities was 58.5\% (439/750); 